\title{
Cartilha Digital 3D como auxílio à alfabetização e letramento: desenvolvimento de aplicativo móvel com uso de realidade aumentada
}

\author{
Alessandro Tetsuo More ${ }^{1}$, Gabriel Amarante Pereira ${ }^{1}$, Willian Alexandre da \\ Silva ${ }^{1}$, Luciene Cavalcanti Rodrigues ${ }^{1,2}$, Naiara Luchini de Assis Kaimoti ${ }^{1}$, Ana \\ Paula Garrido de Queiroga ${ }^{3}$ \\ ${ }^{1}$ Instituto de Federal de Educação, Ciência e Tecnologia de São Paulo (IFSP) \\ Campus Votuporanga \\ Av. Jerônimo Figueira da Costa, 3014 - Pozzobon - Votuporanga, SP - Brasil \\ Grupo de Pesquisa CNPq: Tecnologias e práticas inovadoras aplicadas ao ensino \\ ${ }^{2}$ Faculdade de Tecnologia de São José do Rio Preto (Fatec) \\ Centro Estadual de Educação Tecnológica Paula Souza \\ Rua Fernandópolis, 2510 - Eldorado - S. J. Rio Preto, SP - Brasil \\ ${ }^{3}$ União das Faculdades dos Grandes Lagos - UNILAGO \\ alemore67@gmail.com, prof.luciene@ifsp.edu.br, naiara@ifsp.edu.br, \\ anaproj.eng@gmail.com
}

\begin{abstract}
The reality of education is increasingly linked to technology and thus augmented reality (AR) is not new, despite its great potential is still underused as supporting disciplines and even in the literacy process, and can exemplify easily functions and concepts that were only transmitted through pencil and paper. For this project 3D figures were built with Sketchup tools, Vuforia, Blender and Unity $3 D$, which let you create reality applications increased for varied purposes, whether in creating letters, numbers, images, geometric solids, biology for animations, geography, physical or to demonstrate complex structures in 3D, making the most interactive and dynamic lessons.
\end{abstract}

Resumo. A realidade da educação está cada vez mais ligada a tecnologia e deste modo a realidade aumentada (RA) não é novidade, apesar de seu grande potencial ainda é pouco utilizada como apoio a disciplinas e mesmo no processo de letramento, sendo possível exemplificar de modo simples funções e conceitos que eram apenas transmitidos por meio de lápis e papel. Para este projeto foram construidas figuras $3 D$ com as ferramentas Sketchup, Vuforia, Blender e Unity3D, que permitem criar aplicativos de realidade aumentada para fins variados, seja na criação de letras, números, imagens, sólidos geométricos, animações para biologia, geografia, física ou até demonstrar estruturas complexas em $3 D$, tornando as aulas mais interativas e dinâmicas. 
V Congresso Brasileiro de Informática na Educação (CBIE 2016)

Anais do XXII Workshop de Informática na Escola (WIE 2016)

\section{Introdução}

A Realidade Virtual (RV) é, antes de tudo, uma "interface avançada do usuário" para acessar aplicações executadas no computador ou em dispositivos móveis, tendo como características, a visualização e movimentação em ambientes tridimensionais em tempo real e a interação com elementos do mesmo (Tori, Kirner e Siscoutto 2006 apud. Silva, 2010). Relacionado com a Realidade Virtual, a Realidade Aumentada (RA) é a inserção de objetos virtuais no mundo real por meio de um dispositivo computacional. Desta forma, a interface do usuário é aquela, que se usa no ambiente real, adaptada para visualizar e manipular os objetos virtuais colocados no seu espaço físico (Azuma et al 2001, apud. Silva et al., 2010).

O cerne deste projeto é a fusão de ferramentas de realidade aumentada com a educação propriamente dita, sendo a RA uma complementação ao que está sendo ministrado durante a aula, seja para demostrar o funcionamento de sistemas solares, ou formas sólidas como também para exemplificar conceitos básicos como letras, números, palavras, operações matemáticas, aduzir cálculos em física utilizando animações ou com interação própria do aluno e/ ou professor. Desta forma, foi desenvolvido um aplicativo para a plataforma Android juntamente com uma cartilha, intitulada: "Cartilha Digital 3D - Alfabetização e Letramento: Aprenda Brincando", que poderá ser utilizada livremente por professores e alunos durante o processo de alfabetização e letramento de crianças e adultos, visando proporcionar aprendizagem significativa, representacional e construtivista.

\section{Realidade Aumentada}

De acordo com Erlansari, Santosa e Ferdiana (2013) a Realidade Aumentada (RA) refere-se à exibição objetos ao vivo no mundo real, onde as imagens aumentadas geradas pelo aplicativo são mescladas com o ambiente real, é como a amplificação da percepção sensorial por meio de recursos computacionais. Assim, associando dados computacionais ao mundo real, a Realidade Aumentada permite uma interface mais natural com dados e imagens geradas por computador (Cardoso et al. 2007, apud Silva et al., 2010) que fazem interação com as imagens do mundo real. Desta maneira, entende-se que um sistema de Realidade Aumentada deve prover ao usuário condições de interagir com estes dados de forma mais natural possível. (Silva et al., 2010).

Realidade aumentada tem sido utilizada em várias áreas do conhecimento como ferramenta para auxiliar o processo de ensino-aprendizagem, como, por exemplo, na Matemática, Física, Arquitetura e Construção Civil, Artes, Anatomia, Enfermagem, entre outros.

Com a utilização da RA é possível criar e manipular objetos virtuais que tenham como referência objetos físicos, pessoas ou conteúdos específicos que se deseja demonstrar. Na grande maioria dos casos são utilizados $Q R$ codes (que são imagens assimétricas compostas de símbolos que contém dados) ou no caso deste artigo, simples imagens que funcionam como TAGs para a identificação dos objetos virtuais. Também é possível utilizar os sensores dos dispositivos móveis, tais como smartphones para interação dos objetos com o ambiente, pois com os movimentos do celular também é 
possível trazer múltiplas funcionalidades e interatividade, como por exemplo um objeto virtual que apresente o sistema solar, cada planeta é representado por uma carta de $Q R$ code sendo possível girá-las ou mudá-las de posição caso seja desejado, ou caso seja necessário apresentar como ocorrem os eclipses, podendo ser realizado o posicionado do sol, da terra e da lua, bem como outros conceitos mais simples como dia, noite, estações do ano, translação e rotação dos planetas.

\section{Tecnologia e Educação Pedagógica}

Segundo Ferreira (2009) existem duas maneiras de se entender alfabetização: um processo de aquisição individual de habilidades voltadas para a leitura e escrita, ou um processo de representação de objetos diversos, de naturezas diferentes.

A inserção das novas tecnologias nas relações de ensino-aprendizagem traz inúmeras possibilidades e modifica profundamente as relações com o conhecimento. As características tecnológicas afetam o design instrucional e a maneira de ensinar. Assim, essas novas mídias alteram a experiência de aprendizagem (Kato et al. 2014, apud Silva, Reis, Lima e Teichrieb, 2014).

Segundo Borba et al. (2001), a tecnologia é desenvolvida para suprir as necessidades do nosso cotidiano tal como: pesquisas, método ou processo de desenvolvimento e produção, entre outras. Mas sempre pensando no bem-estar ou uma maneira mais rápida de se elaborar algo. Sendo ela também presente na área da educação podendo estimular cada vez mais os alunos a procurar novos recursos e aprendizados, de uma maneira mais fácil e atrativa. A tecnologia está no nosso dia-a-dia principalmente nos últimos anos no qual está presente em praticamente tudo a nossa volta.

Conforme diz Valente (1993), o computador é um meio didático: assim como temos o retroprojetor, o vídeo, etc., devemos ter o computador. Nesse caso o computador é utilizado para demonstrar um fenômeno ou um conceito, antes do fenômeno ou conceito ser passado ao aluno. De fato, certas características do computador como capacidade de animação, facilidade de simular fenômenos, contribuem para que ele seja facilmente usado na condição de meio didático. No entanto, isso pode ser caracterizado como uma subutilização do computador se pensarmos nos recursos que ele oferece como ferramenta de aprendizagem.

\section{Ferramentas Tecnológicas: Vuforia, Unity3D, Blender}

O Vuforia é uma engine de criação de elementos fundamentais para a criação de objetos em realidade aumentada, desenvolvida pela Qualcomm Developer Network. Consiste de um pacote de desenvolvimento de softwares de realidade aumentada para dispositivos móveis assim permite a criação de aplicativo de realidade aumentada. Este usa a tecnologia de visão computacional para reconhecer e localizar, em tempo real, imagens planas (Image Targets) e objetos simples, como caixas. Pode ser utilizada em conjunto com o Unity3 $\mathrm{D}$ e assim podendo ser moldada em linguagens como $\mathrm{C}++$, Java e .Net ([s.a.], 2016). 
V Congresso Brasileiro de Informática na Educação (CBIE 2016)

Anais do XXII Workshop de Informática na Escola (WIE 2016)

O Unity3D é uma plataforma de criação de jogos desenvolvida pela Unity Technologies, usada para desenvolver jogos para computador, consoles, dispositivos moveis e websites ([s.a.], 2016).

O Sketchup é uma ferramenta criação de imagens 3D, muito utilizada por arquitetos e engenheiros pois integra desenhos 2D e 3D. O Blender também conhecido como Blender3D é um programa de modelagem, animação, texturização, composição, renderização, edição de aplicações interativas e de vídeo, assim como jogos. $\mathrm{O}$ blender3D é Open source, ou seja, este é em código aberto e totalmente gratuito para a aquisição.

Estes quatro aplicativos foram utilizados para a criação do projeto, na criação de qualquer tipo de aplicativo usando o Vuforia primeiramente é necessário criar uma conta no site do mesmo, após a criação da conta é necessário baixar o plugin do Vuforia para o Unity3 $\mathrm{D}^{1}$. Qualquer Image Target usada é criada no próprio site do Vuforia, para cria-lo primeiramente se cria uma licença, após criada é adicionada a imagem desejada e para que esta seja importada para o Unity3D é preciso realizar o download do Banco de Dados criado com as Image Targets ${ }^{2}$. Dentro do Unity3D a primeira coisa a ser feita é importar a extensão do Vuforia e o Banco de dados contendo as Image Targets, após ter realizado estas ações é necessário excluir todos os itens padrões adicionados ao pacote, tais como a Main Camera. Para a Cartilha Digital 3D foram criadas várias páginas contendo capa, introdução, pequenas explicações a respeito de vogais e números e as respectivas Image Targets, que são a base para a leitura e animação pelo aplicativo.
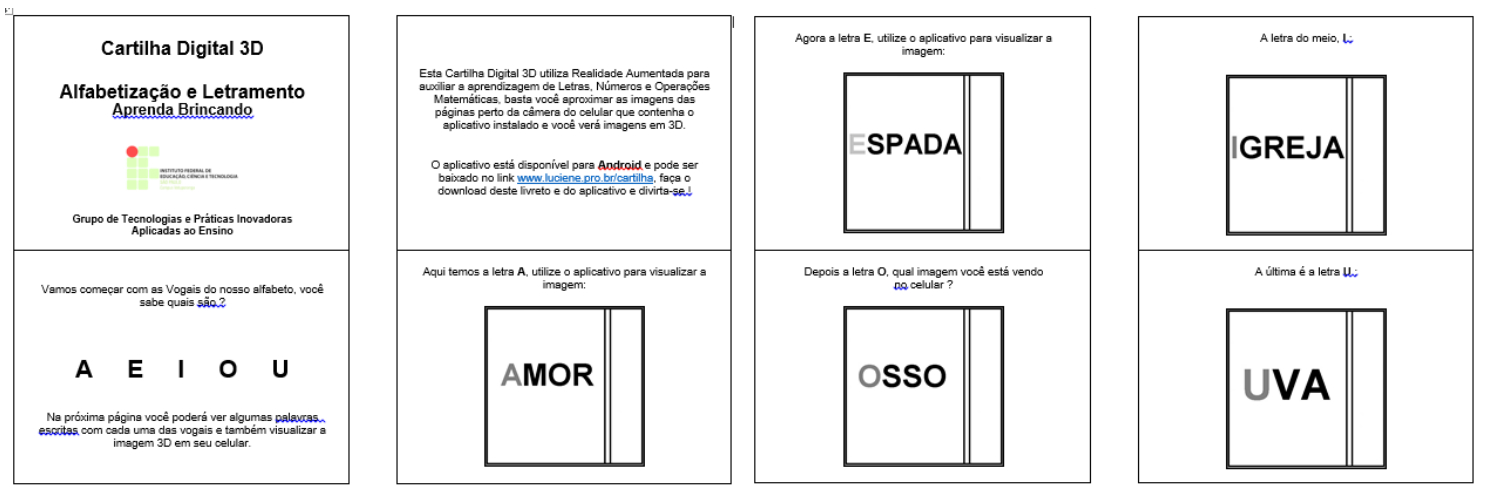

Figura1. Cartilha Digital 3D: Apresentação e conceitos sobre vogais

No tocante aos números (Figura 2), foram trabalhados os números de 0 a 10 , bem como as operações aritméticas básicas, criando um pequeno jogo de animação que pergunta o resultado das operações.

\footnotetext{
${ }^{1} \mathrm{https}: / /$ developer.vuforia.com//downloads/sdk

${ }^{2}$ https://developer.vuforia.com/target-manager
} 
V Congresso Brasileiro de Informática na Educação (CBIE 2016)

Anais do XXII Workshop de Informática na Escola (WIE 2016)
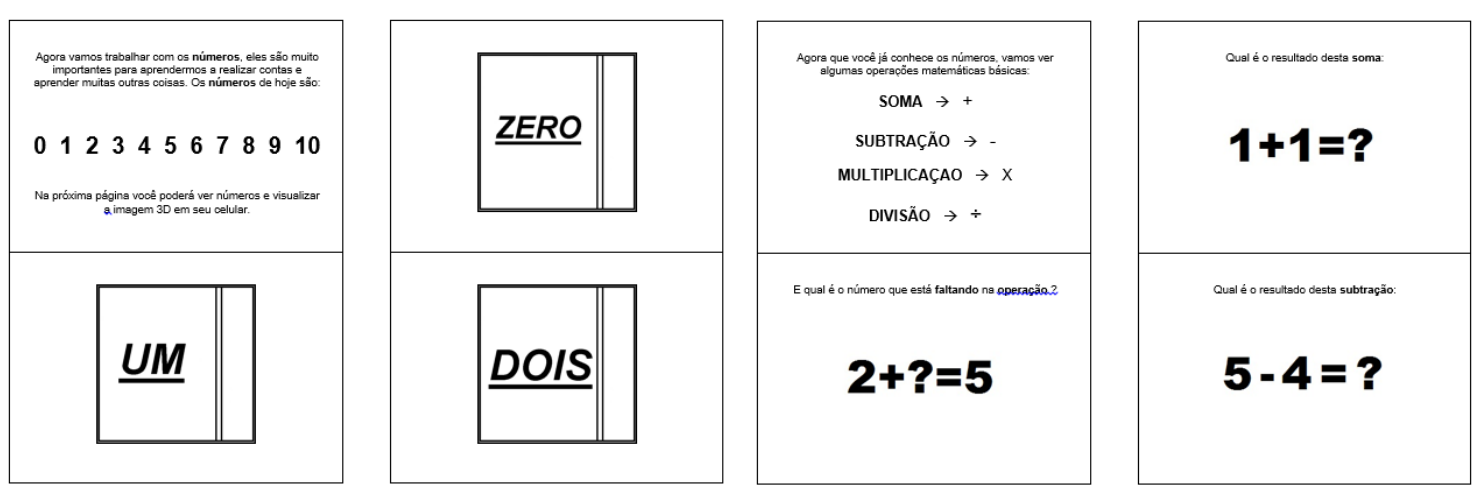

Figura 2. Cartilha Digital 3D: Números e operações aritméticas

\section{Resultados}

Com o auxílio dos aplicativos mencionados foi produzido o aplicativo com o objetivo principal de demonstrar as vogais e seu uso em algumas palavras, os números e suas operações aritméticas básicas, afim de auxiliar a apresentação para os alunos dos mesmos.

Na figura 3 são mostradas as Image Target, que são as representações impressas dos objetos e com o uso do aplicativo, como o mesmo é apresentado aos alunos por meio do dispositivo móvel. Desta forma os alunos poderão visualizar os objetos no celular ou no projetor com lousa digital para visualizar as letras, palavras ou números ao mesmo tempo que o professor explica suas características.

Ao posicionar a câmera da cartilha com o aplicativo aberto será possível visualizar um número em cada página. Na Cartilha está escrito por extenso o nome do número, já no aplicativo é exibido o numeral (Figura 3).

Após a apresentação dos números são apresentadas algumas operações aritméticas simples no formato de adivinhação, apresentando uma interrogação no lugar do número a ser descoberto, ao posicionar a página da câmera será exibido o valor que está faltando (Figura 5). Em relação às vogais, são apresentadas palavras com cada vogal, ao posicionar a página da câmera do aplicativo é exibida uma imagem referente à palavra, a letra inicial e a palavra reescrita (Figura 6). 
V Congresso Brasileiro de Informática na Educação (CBIE 2016)

Anais do XXII Workshop de Informática na Escola (WIE 2016)
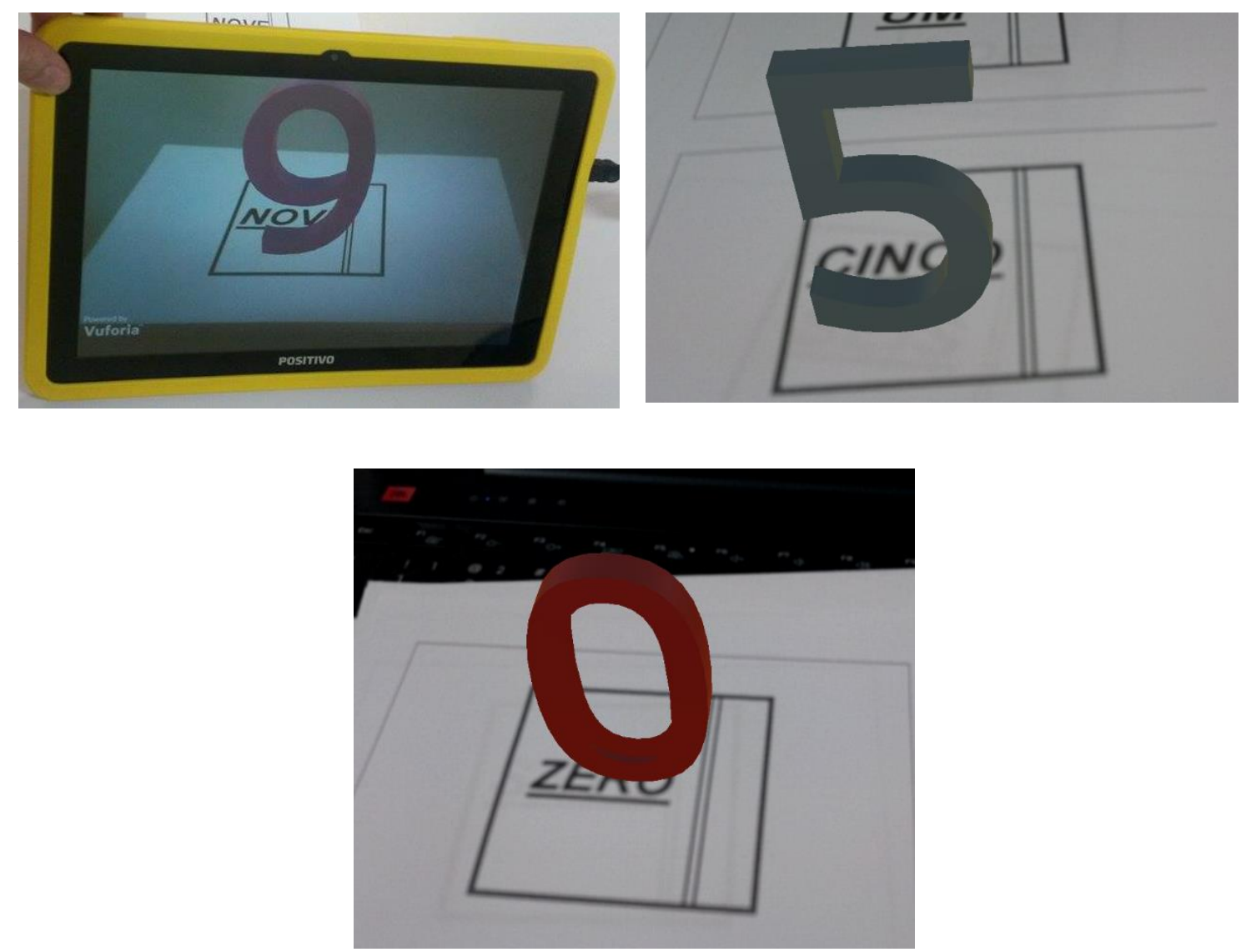

Figura 3. Números
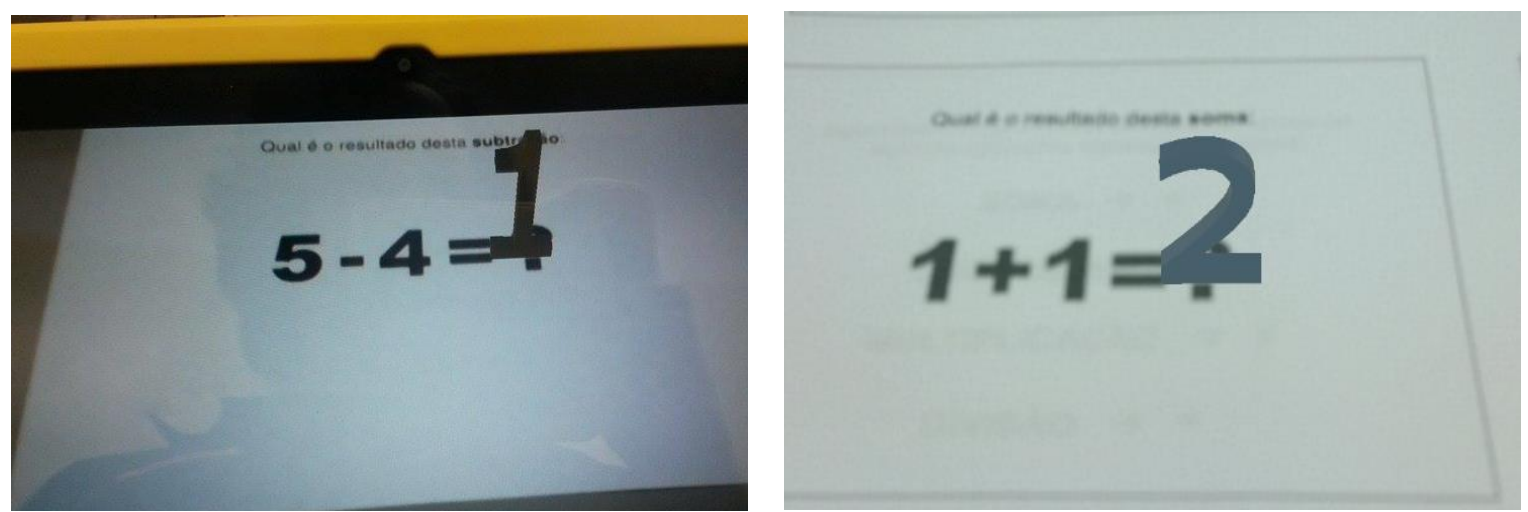

Figura 4. Operações Aritméticas

As imagens apresentadas são todas coloridas e diferenciadas umas das outras, visando trazer novidade a cada exibição. Também é possível colocar exibições com imagens ou animações junto ao conteúdo, porém nesta versão optou-se por deixar a exibição limpa e simples, visto que é voltado para crianças e fase de letramento e início da alfabetização. 
V Congresso Brasileiro de Informática na Educação (CBIE 2016)

Anais do XXII Workshop de Informática na Escola (WIE 2016)
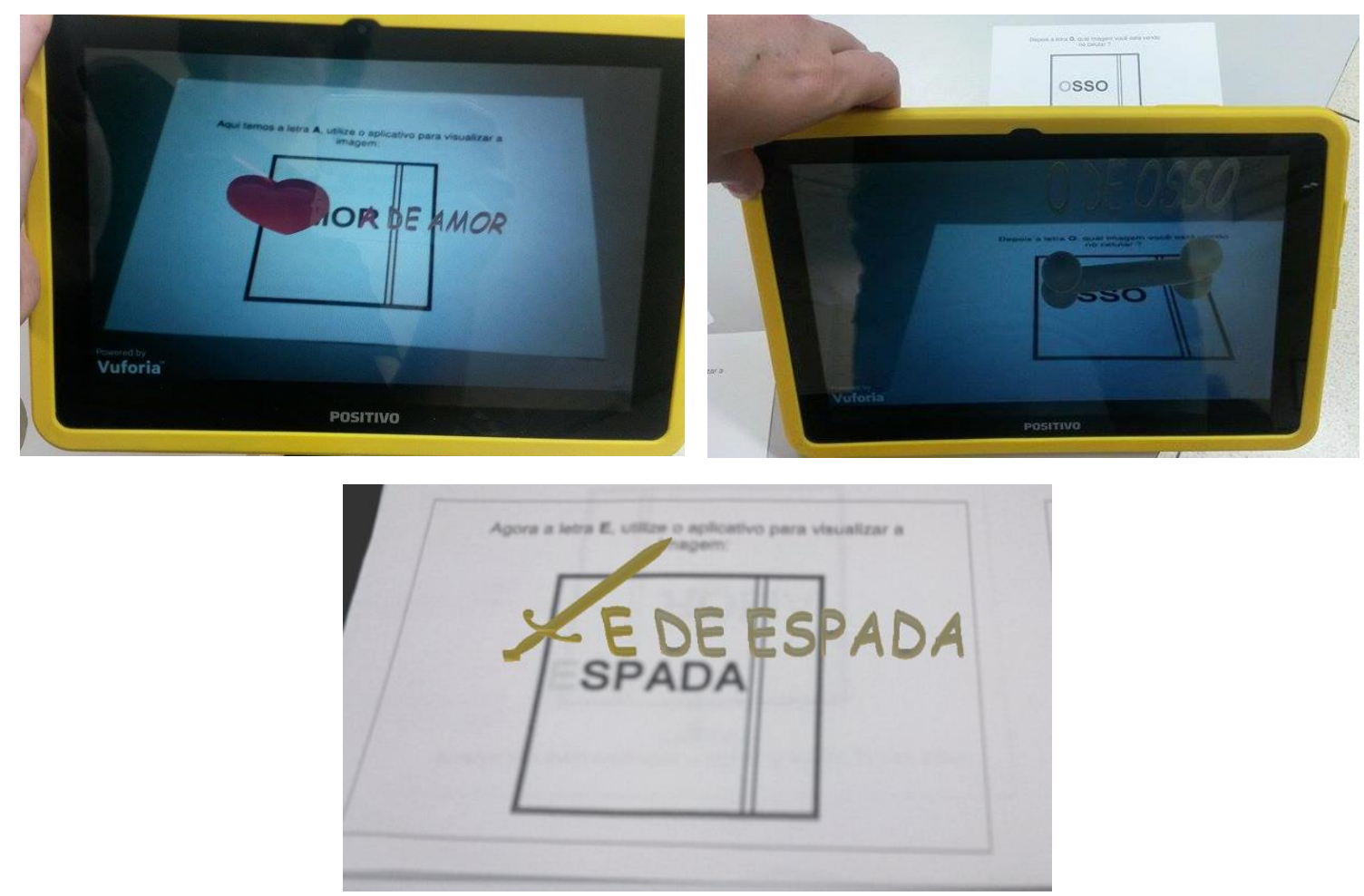

Figura 5. Vogais e palavras

\section{Conclusão}

Como foi apresentado, a realidade aumentada possui diversas finalidades, fáceis de se trabalhar, pois este possui diversos meios de utilização, seja jogos, animações, apresentação de formas ou imagens em 3D, a realidade aumentada está sendo espalhada nos setores não apenas da educação, como no entretenimento e em áreas industriais.

A cartilha está disponibilizada no formato pdf em versão para impressão frente e verso ou só frente. Visto que as páginas da cartilha possuem o tamanho A5, serão impressas 4 páginas por folha $\mathrm{A} 4$, necessitando então que sejam cortadas e organizadas de acordo com o uso e necessidade dos professores. O aplicativo também está disponível em http://luciene.pro.br/?p=21 e pode ser instalado em qualquer dispositivo Android que possua uma câmera. Foram realizados testes de instalação e uso em diferentes dispositivos, como celulares mais atuais, celulares com pouca capacidade de processamento e tablets, todos foram capazes de executar a aplicação com sucesso, a única diferença perceptível foi no carregamento da aplicação para uso que é mais lenta em dispositivos com pouco processamento. Quanto à capacidade de armazenamento, o aplicativo necessita de pouco menos de $20 \mathrm{Mb}$ de espaço para instalação, visto que o aplicativo possui muitas imagens a serem processadas, acarreta um tamanho maior para a aplicação final.

Este projeto foi apresentado aos alunos e professores do ensino fundamental I da cidade de Votuporanga durante uma visita ao campus do Instituto Federal de Educação, 
Ciência e Tecnologia da mesma cidade e segundo entrevistas foi possível verificar que a usabilidade foi agradável e a motivação de todos foi surpreendente, formando fila para a visualização das imagens em 3D. Não foram realizados questionários de avaliação do projeto em sala de aula, mas em relação à pesquisa com os visitantes (cerca de 12 professores) todos responderam que estariam dispostos a utilizar o aplicativo em sala de aula, bem como várias crianças levaram para casa as cartilhas e disseram que iriam pedir aos pais para instalar e utilizar.

Em sua próxima fase, a cartilha trabalhará conceitos sobre cores, figuras geométricas e interatividade dos alunos para a criação de jogos utilizando realidade aumentada. Também já estão agendadas visitas às escolas para apresentação do aplicativo e da cartilha aos professores do ensino fundamental I das escolas municipais de Votuporanga.

\section{Referências}

[s.a] (2016). Vuforia Getting Started. Disponível em: $<$ https://library.vuforia.com/getting-started $>$ Acesso em: 10/08/2016.

[s.a.] (2016). Unity Manual. Disponível em: $<$ http://docs.unity3d.com/Manual/index.html> Acesso em: 10/08/2016.

BORBA, Marcelo de Carvalho; PENTEADO, Miriam Godoy. (2001) "Informática na Educação." Belo Horizonte: Autêntica, 2001. Disponível em: <http://nteestrela.pbworks.com/f/Fabr\%C3\%ADcio++Inform\%C3\%A1tica+na+Educa\%C3\%A $7 \% \mathrm{C} 3 \% \mathrm{~A} 3 \mathrm{o} . \mathrm{doc}>$.

ERLANSARI, A.; SANTOSA, P. I., FERDIANA, R. (2013). Augmented Reality Application for Book Promotion . International Conference on Information Systems for Business Competitiveness (ICISBC 2013).

FERREIRA, Dalva Aparecida et al. (2009) "Letramento: Repensando o ensino da língua escrita." Disponivel em: < http://www.unisalesiano.edu.br/encontro2009/trabalho/aceitos/CC35128563812.pdf $>$.

LENDER.ORG. (2015) “Blender.” Disponível em: <https://www.blender.org/about/>.

MARTINS, Valéria F. et al. (2014) "PC-AR : Apoio ao Ensino de Organização de Computadores utilizando Realidade Aumentada." 2014. 25o. WIE. CBIE 2014 Disponível em: <http://www.br-ie.org/pub/index.php/sbie/article/view/2945/2679>.

SANTOS, E. T.; MARTINEZ, M. L. (2000) "Software para ensino de geometria e desenho técnico." Ouro Preto: Graphica. Disponível em:< http://www.researchgate.net/profile/Eduardo_Santos6/publication/237359480_SOFT WARE_PARA_ENSINO_DE_GEOMETRIA_E_DESENHO_TCNICO/links/02e7e 536a48e7102d4000000.pdf $>$.

SILVA, Manoela et al. (2014) "ARJigsawPuzzle: PotencialidadesdeUsodaRealidade AumentadanoEnsinodeGeografia." 2014. 25o. WIE. CBIE 2014 Disponível em: $<$ http://www.br-ie.org/pub/index.php/wie/article/view/2041/1803>. 
V Congresso Brasileiro de Informática na Educação (CBIE 2016)

Anais do XXII Workshop de Informática na Escola (WIE 2016)

SILVA, Wender Antônio da et al. (2010) "Ambientes Interativos e Colaborativos baseados em Realidade Aumentada aplicados à Educação." 2010. 210. WIE. CBIE $2014 \quad$ Disponível em: <http://www.brie.org/pub/index.php/wie/article/view/2041/1803>. Acesso em: 12 maio 2015.

VALENTE, José Armando. (1993) "Por quê o computador na educação. Computadores e conhecimento: repensando a educação. Campinas: Unicamp/Nied”, p. 24-44, 1993. Disponivel em: http://xa.yimg.com/kq/groups/23266122/1283528405/name/Por+Qu\%C3\%AA+o+C omputador+na+Educa\%C3\%A7\%C3\%A3o.pdf $>$

VALENTE, J. A. et al. (1993), "Diferentes usos do computador na educação. Computadores e Conhecimento: repensando a educação", p. 1-23, 1993. Disponível em: <http://ffalm.br/gied/site/artigos/diferentesusoscomputador.pdf> 